\title{
Erratum to: Internal tibial rotation during in vivo, dynamic activity induces greater sliding of tibio-femoral joint contact on the medial compartment
}

\author{
Yuichi Hoshino $\cdot$ Scott Tashman
}

Received: 12 September 2012/ Accepted: 22 October 2012/Published online: 6 November 2012

(c) Springer-Verlag Berlin Heidelberg 2012

Erratum to: Knee Surg Sports Traumatol Arthrosc

(2012) 20:1268-1275

DOI 10.1007/s00167-011-1731-6

We have identified an error in one of the formulas printed in the manuscript. The erroneous and corrected formulas are as follows:

Formula printed in the paper (page 1270, right column, line 10):

Femoral contact path

$$
=\sum \sqrt{R_{n}^{2}+R_{n+1}^{2}-R_{n} R_{n+1} \cos \left(J_{n+1}-J_{n}\right)^{2}}
$$

Correct formula:

Femoral contact path

$$
=\sum \sqrt{R_{n}^{2}+R_{n+1}^{2}-R_{n} R_{n+1} * 2 * \cos \left(J_{n+1}-J_{n}\right)}
$$

The error was due to a mistake made when the formula was typeset during manuscript preparation. The correct formula was used for the data analysis. Therefore, the data, results and conclusions of the work are not affected.

The online version of the original article can be found under doi:10.1007/s00167-011-1731-6.

Y. Hoshino $\cdot$ S. Tashman $(\bowtie)$

Department of Orthopaedic Surgery, Orthopaedic Research Laboratories, University of Pittsburgh, 3820 South Water St., Pittsburgh, PA 15203, USA

e-mail: tashman@pitt.edu 